\title{
Drying mechanism of monodisperse colloidal film: Evolution of normal stress and its correlation with microstructure
}

\author{
JAE HWAN JEONG${ }^{1}$, Young Ki Lee ${ }^{1}$, and Kyung Ahn ${ }^{1}$ \\ ${ }^{1}$ Seoul National University
}

March 12, 2021

\begin{abstract}
We investigate the drying process of monodisperse colloidal film over a wide range of Péclet number (Pe) by using the Brownian dynamics simulation. We analyze the detailed process in three aspects; accumulation front, normal stress, and microstructure. The evolution of particle distribution is quantified by tracking the accumulation front. The accumulated particles contribute to the continuous increase of the normal stress at the interface. At the substrate, the normal stress first stays constant and then increases as the accumulation front touches the substrate. We quantitatively analyze the stress development by a scaled normal stress difference between the two boundaries. At all tested Pe, the stress difference increases to the maximum, followed by a decrease during drying. Interestingly, a mismatch is observed between the stress difference maximum and the initial stress increase at the substrate. The microstructural analysis reveals that this mismatch is related to the microstructural development at the substrate.
\end{abstract}

\section{Hosted file}

Manuscript_Drying mechanism of monodisperse colloidal film.pdf available at https: //authorea.com/users/401208/articles/513378-drying-mechanism-of-monodisperse-colloidalfilm-evolution-of-normal-stress-and-its-correlation-with-microstructure

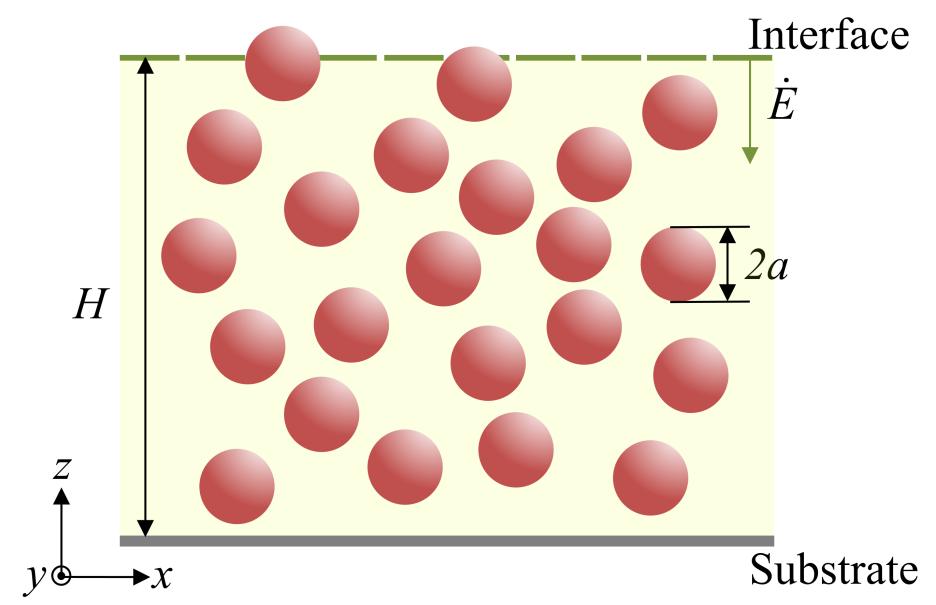

Figure 1. Schematic diagram of the monodisperse colloidal film drying. 
(a)

$H / H_{0}$

0.8

0.6

0.4

0.3

0.2
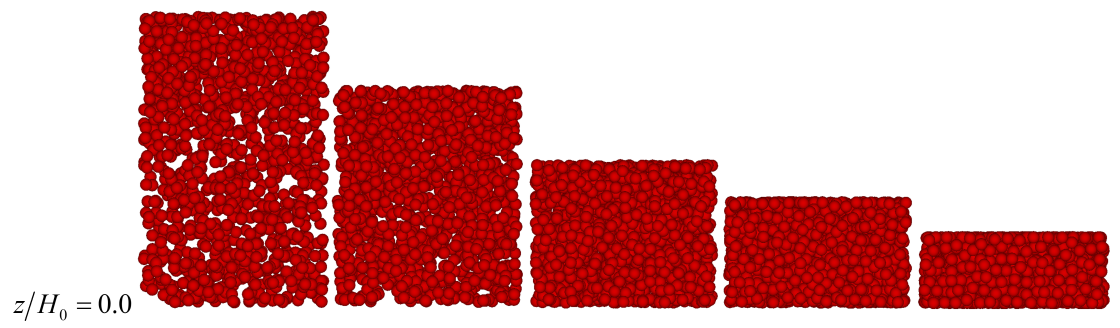

(b)

$H / H_{0} \quad 0.8$

0.6

0.4

0.3

0.2
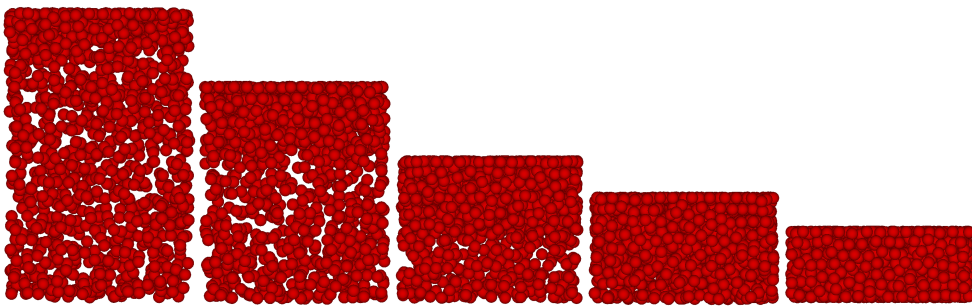

Figure 2. Time evolution of particle configuration in drying film. (a) $P e=6$; (b) $P e=60$.

(a)

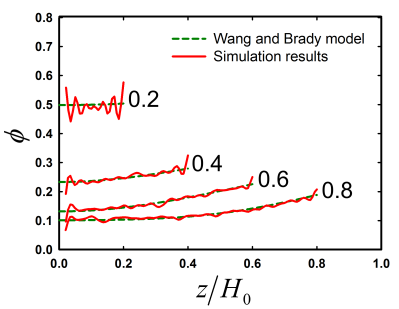

(b)

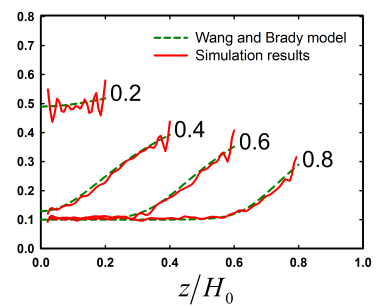

(c)

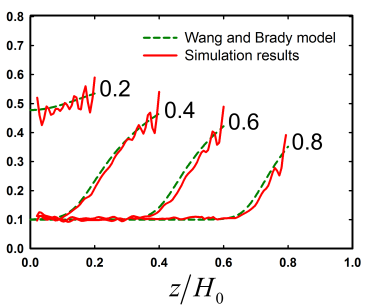

Figure 3. Local volume fraction profile with the film thickness during drying. (a) $P e=6$; (b) $P e=30$; (c) $P e=60$. The volume fraction in the model of Wang and Brady is the green dashed line, and the simulation the red solid line. $H / H_{0}$ is denoted next to the corresponding profile. 


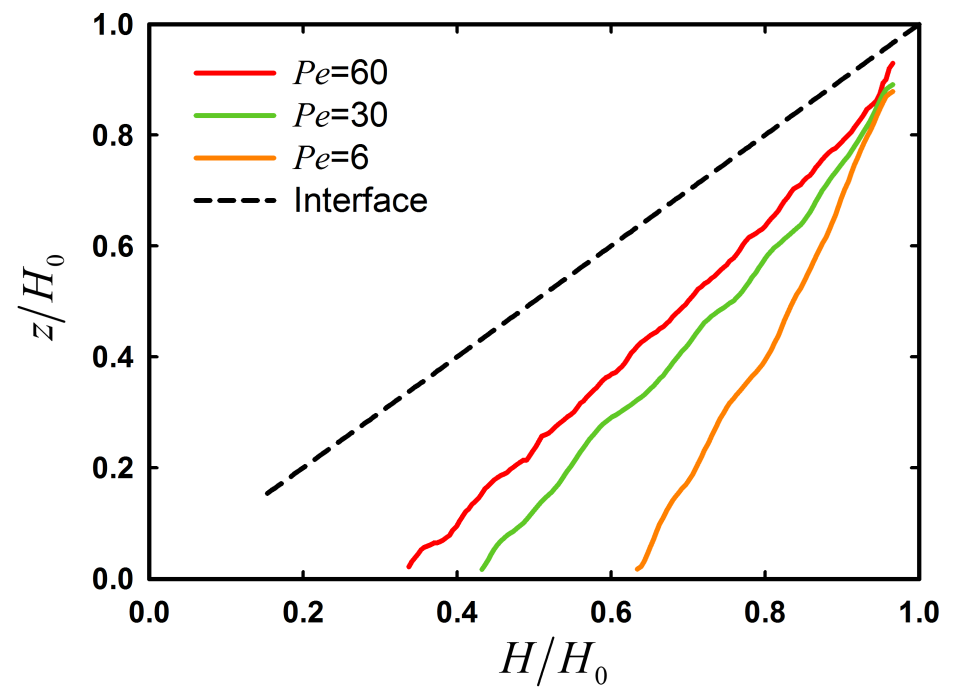

Figure 4. The position $z$ of the accumulation front with the film thickness.

(a)

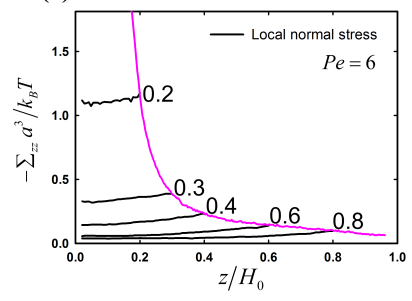

(b)

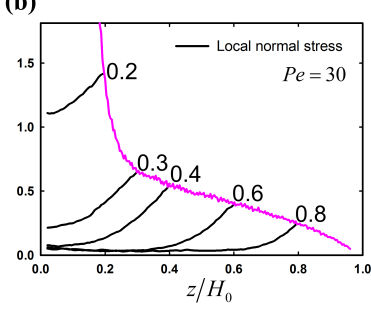

(c)

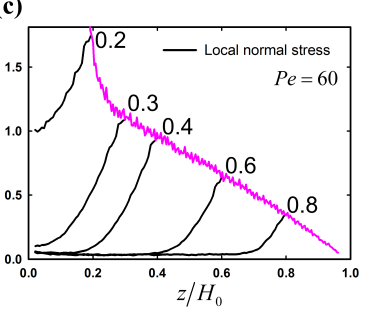

Figure 5. Local normal stress profile with the film thickness during drying. (a) $P e=6$; (b) $P e=30$; (c) $P e=60$. The black solid line represents the local normal stress profile at a given film thickness, and the pink solid line represents the normal stress at the interface over time (calculated by extrapolating to the position of the interface using the local normal stress value near the interface).

(a)

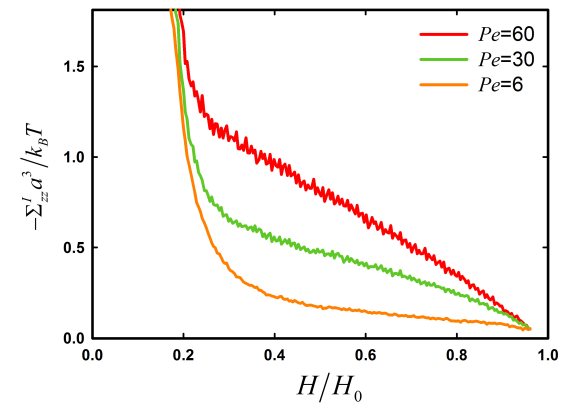

(b)

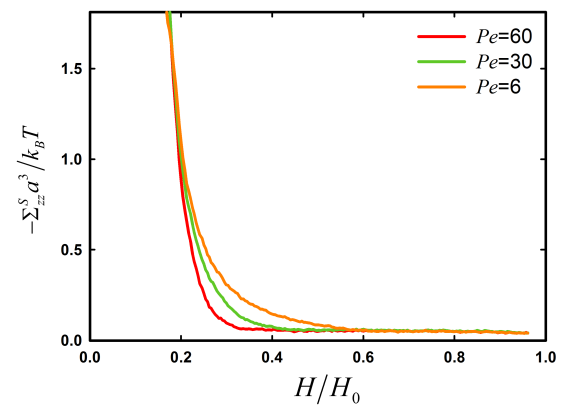

Figure 6. Development of local normal stress at the (a) interface $(z=H)$ and (b) substrate $(z=a)$. 


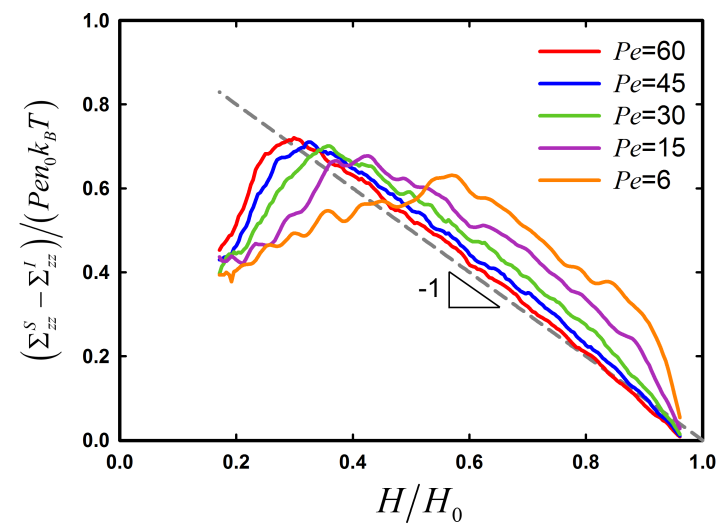

Figure 7. The scaled normal stress difference between the interface and substrate with the film thickness. The gray dotted line represents a guideline with a slope of -1 .

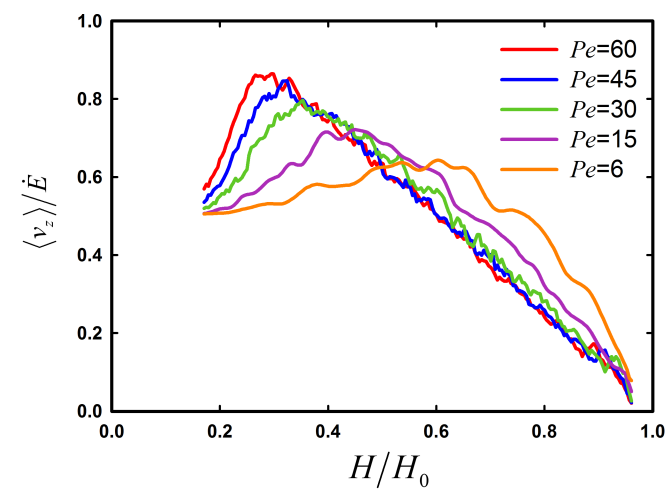

Figure 8. Normalized average particle velocity in the $z$-direction with the film thickness. Note that the evaporation rate has a (-) sign as the interface moves down to the $-z$ direction.

(a)
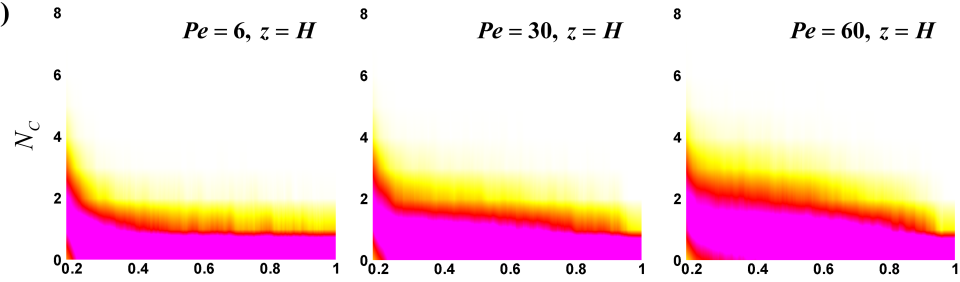

$P\left(z, N_{C}\right)$
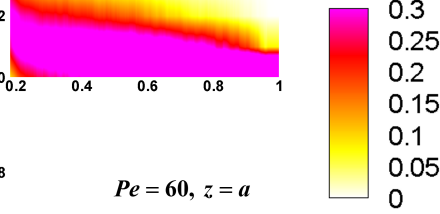

(b) 8
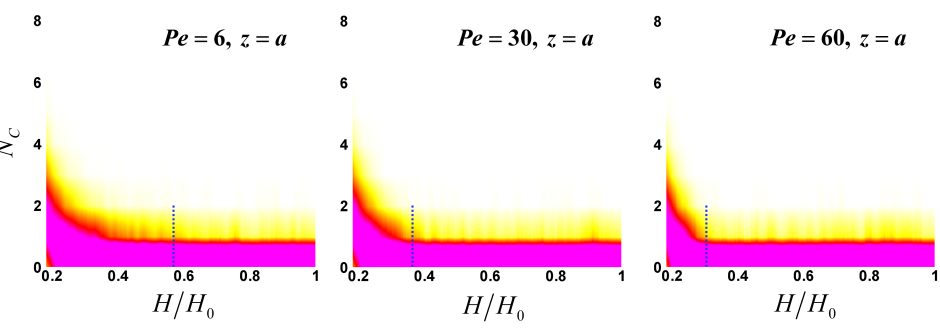

Figure 9. Average contact number distribution of particles $P\left(z, N_{C}\right)$ at the interface and substrate with film thickness. (a) interface $(z=H)$; (b) substrate $(z=a)$. The blue dotted line represents the film thickness of the normal stress difference maximum (see Figure 7). 

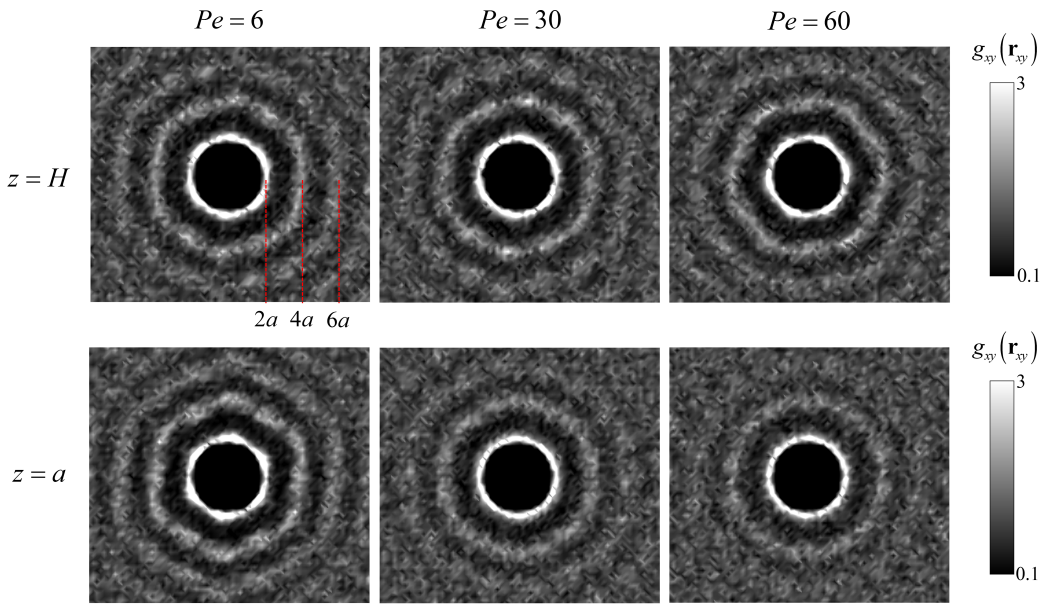

Figure 10. 2D planar pair-distribution function $g_{x y}\left(\mathbf{r}_{x y}\right)$ (at $\left.H / H_{0}=0.18\right)$ at the interface $(z=H)$ and substrate $(z=a)$ Red dotted lines represent $r_{x y}=2 a, 4 a$, and $6 a$ from the left, respectively. 